Research Article

\title{
Experimental Investigation on Influencing Factors of Rock Fragmentation Induced by Carbon Dioxide Phase Transition Fracturing
}

\author{
Bo Gao, ${ }^{1}$ Youjiang Yang, ${ }^{1}$ Weilong Xue, ${ }^{1}$ Anhui Guo, ${ }^{1}$ and Xuedong Luo $\mathbb{D}^{2}$ \\ ${ }^{1}$ CCCC Second Highway Consultants Co., Ltd., Wuhan 430056, China \\ ${ }^{2}$ Faculty of Engineering, China University of Geosciences, Wuhan 430074, China \\ Correspondence should be addressed to Xuedong Luo; luoxd@cug.edu.cn
}

Received 5 January 2021; Revised 23 January 2021; Accepted 22 August 2021; Published 7 September 2021

Academic Editor: Roman Gabl

Copyright (C) 2021 Bo Gao et al. This is an open access article distributed under the Creative Commons Attribution License, which permits unrestricted use, distribution, and reproduction in any medium, provided the original work is properly cited.

Carbon dioxide phase transition fracturing is a novel physical blasting technique, which is gradually used in mining and underground space engineering. The improvement of its rock breaking efficiency is the key concern in the application. In this paper, field experiments of $\mathrm{CO}_{2}$ phase transition fracturing were conducted. Based on the strain monitoring and fracturing crater volume measuring, the variation of $\mathrm{CO}_{2}$ filling amount and shear sheet thickness on rock fragmentation of $\mathrm{CO}_{2}$ phase transition fracturing was investigated. The experimental results indicated that the fracturing crater is shaped as an elliptical cone that is longer in the jet direction and shorter in the vertical jet direction. With the increase of the $\mathrm{CO}_{2}$ filling amount, the excavated crater volume gradually increases, but the growth rate gradually decreases. The powder factor is constant within a certain charge amount, and after exceeding this charge amount, the powder factor of $\mathrm{CO}_{2}$ increases significantly. As the shear sheet thickness increases, although the consultant peak stress gradually increases, its growth rate is still unchanged. The crater volume and its growth rate gradually increase in the same situation. Moreover, with the shear sheet thickness increase, the $\mathrm{CO}_{2}$ powder factor decreases continuously, and the decline rate remains unchanged.

\section{Introduction}

Due to the harmful effects like blasting seismic waves, noise, flying rocks, and harmful gases, the use of explosives in inflammable and combustible, sensitive areas is limited $[1,2]$. Therefore, there is an urgent need for a safe, environmentally friendly, and efficient nonexplosive rock breaking method. As a new type of physical rock breaking technology, $\mathrm{CO}_{2}$ phase transition fracturing breaks the rock mass by the expansion energy of $\mathrm{CO}_{2}$ liquid-gas phase transition. The whole fracturing process is nonsparking, and the phase transition product is a flame-retardant harmless gas $[3,4]$. In general, its safety and environmental protection are far better than blasting, and this technique has a broad application prospect as an alternative technology for blasting.

In 1914, the British company Cardox developed a system for the permeability and recovery of low-breathing and high-gas coal seams, reducing coal dust and reducing the gas explosion risk. And it is called the Cardox tube system. Shortly hereafter, Cardox tube system was widely used for coal mining in the United States, Germany, Britain, France, and other countries. Due to the rapid development of coal mining technology and mechanized production of coal mines, coal mines gradually stopped using the Cardox tube system. In recent years, $\mathrm{CO}_{2}$ phase transition fracturing has been widely used in the field of rock excavation due to its multiple advantages [5], such as pile-well excavation [6], gas drainage [7-9], coal roadway excavation [10,11], and coal mining face mining [12].

Rock fragmentation is essential for evaluating a new rock excavation method. Through the tests in Bulawayo gold mine, Singh [13] confirmed that the combined detonation effect of two $\mathrm{CO}_{2}$ fracturing pipes was the same as that of five boreholes filled with explosives, indicating that this 
technique was a good rock breaking method. Some scholars have also explored the rock fragmentation with different initial parameters. Zhan [14] investigated the influence of different $\mathrm{CO}_{2}$ filling amounts on the effective damage range in coal through numerical simulation, indicating that damage radius and depth are positively correlated with the filling amount. Zhang et al. $[15,16]$ carried out the fracturing tests on the concrete block, established the peridynamics model to study the evolution of cracks, and indicated that gas impact and gas wedge are both important to rock fragmentation, and the direction of crack propagation was perpendicular to that of carbon dioxide release. Zhou et al. [4] got a similar conclusion with them through the field test and numerical simulation and indicated that the fracturing crack propagation speed decreased with time. Tao et al. [17] compared the rock breaking mechanism between the carbon dioxide phase transition fracturing and blasting and drew the conclusion that there are no crushing zones after $\mathrm{CO}_{2}$ phase transition fracturing; thus, the explosive energy utilization rate is correspondingly improved. Tian [18] investigated the influence of confining pressure on the number and shape of cracks and pointed out that the number of cracks produced by $\mathrm{CO}_{2}$ phase transition fracturing is more than that of hydraulic fracturing.

Previous studies on the influencing factors of rock fragmentation for $\mathrm{CO}_{2}$ phase transition fracturing almost carried out on the rock mass with good quality, complete concrete block and soil, the crack evolution process were investigated. The rock fragmentation characteristics of fractured rock masses which are induced by $\mathrm{CO}_{2}$ phase transition fracturing are not yet clear. Moreover, there are few studies on the influence of initial parameters of fracturing on rock fragmentation characteristics. Through the in situ tests, this paper investigates the influence of $\mathrm{CO}_{2}$ filling amount and shear sheet thickness for rock fragmentation on fractured mudstone, with dynamic strain monitoring and crater volume measurement. The test results may provide the reference for drill hole design on fractured rock masses.

\section{Principle of $\mathrm{CO}_{2}$ Phase Transition-Induced Rock Fragmentation}

$\mathrm{CO}_{2}$ phase transition fracturing pipe consists of a detonator, a heating tube, a liquid storage pipe, a gasket, a shear sheet, and an energy drain head. When we conduct the $\mathrm{CO}_{2}$ fracturing, we should first fill the liquid carbon dioxide into the liquid storage pipe, and then the filled $\mathrm{CO}_{2}$ fracturing pipe is inserted into the predrilled hole with signal line reserved and the hole sealed. Then, the heating tube will be energized by current and immediately release a lot of heat in a short time. While the liquid carbon dioxide absorbs heat and transfers to the supercritical state, the pressure in the storage pipe is continually rising. When the inner pressure of the storage pipe exceeds the shear sheet strength, the shear sheet is broken. Supercritical carbon dioxide quickly transfers to high-pressure gas and releases from the outlet of the energy drain head. At the same time, the high-pressure gas instantly brings the strong destructive force; the surrounding rock in the jet direction is firstly be compressed under the shock stress, resulting in a certain number of cracks. Then, the high-pressure gas enters the impact cracks; these cracks propagate forward again under the highpressure gas wedge $[18,19]$. During this process, the thickness of shear sheet determines the peak compressive stress; the filling amount of carbon dioxide determines the total amount of high-pressure gas and the duration of the gas wedge, both of which have a decisive influence on rock fragments. The rock breaking process for the $\mathrm{CO}_{2}$ phase transition fracturing is shown in Figure 1.

Nowadays, when describing the power of $\mathrm{CO}_{2}$ phase transition fracturing, the $\mathrm{CO}_{2}$ phase transition fracturing is often equivalent to the pressure vessel explosion. When we calculate the physical explosion energy of pressure vessels, the explosion energy calculation method of liquefied gas and high-temperature saturated water container and the explosion energy calculation method of high-pressure gas and saturated water vapor container are widely used. The former method is mainly used to describe superheated liquid, and liquid carbon dioxide is not a superheated liquid; thus, this method is not suitable for this fracturing technique. In contrast, the existence state of carbon dioxide in the liquid storage pipe and the explosion trigger conditions are consistent with the explosion energy calculation method of high-pressure gas and saturated water vapor container, so this method is the most widely recognized. The corresponding equation of this method is given by

$$
E_{g}=\frac{p V}{K-1}\left[1-\left(\frac{0.1013}{p}\right)^{(K-1) / K}\right] \times 10^{3}
$$

where $E_{g}$ is the total explosive energy of the carbon dioxide, $\mathrm{kJ}$; $p$ is the maximum inner pressure of fracturing pipe, $\mathrm{MPa}$; $V$ is the effective volume for the storage pipe, $\mathrm{m}^{3}$; and $K$ is the adiabatic index of carbon dioxide, taken as 1.295.

\section{Effect of Carbon Dioxide Filling Amount on Rock Fragments}

3.1. Field Test. Strong-to-medium weathered mudstone with basic quality grade $V$ was chosen as fracturing medium in this experiment; its uniaxial compressive strength was less than $10 \mathrm{MPa}$, belonging to extremely soft rock. To investigate the effect of carbon dioxide filling amount on rock fragments, five kinds of fracturing pipes with different capacities were chosen, as is shown in Figure 2(a).

During the process of liquid carbon dioxide filling, when the inner pressure in the fracturing pipe reaches a preset value, the filling machine could not press liquid carbon dioxide into the fracturing pipe. At that time, we should remove the fracturing pipe from the filling shelf and measure the mass of filled carbon dioxide. The filling equipment of $\mathrm{CO}_{2}$ fracturing pipe and the platform scale for weighting the fracturing pipe before and after the filling are shown in Figures 2(b) and 2(c).

After drilling, the filled fracturing pipes were put into the drill hole, and medium-coarse sand and small pieces of gravel were chosen as stemming materials to prevent the 


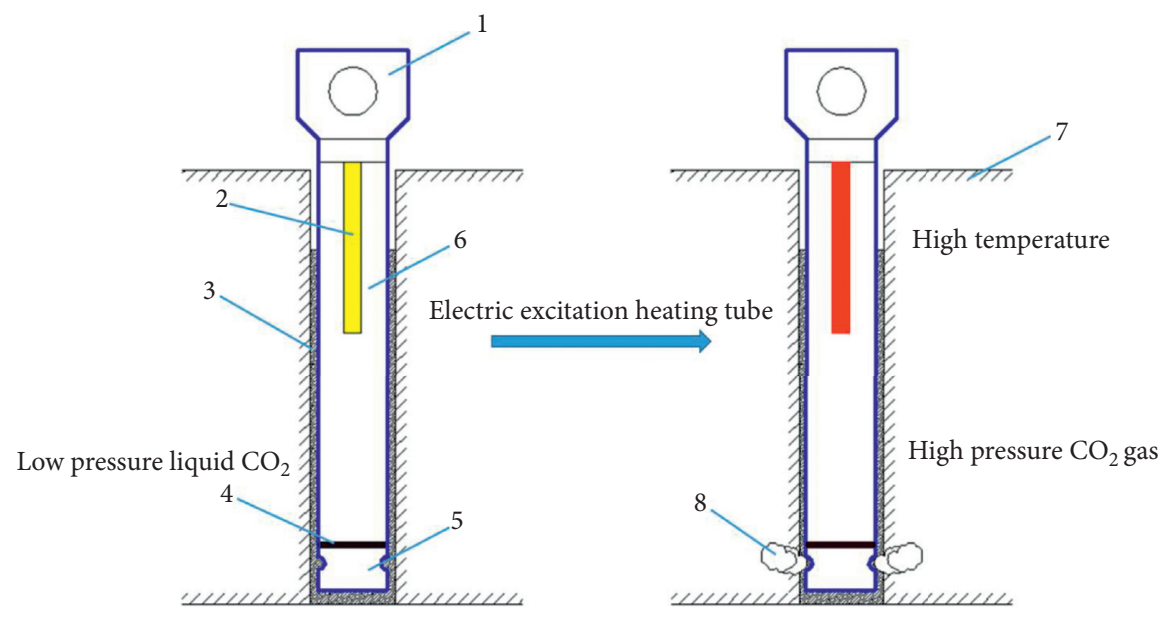

1-draw bar ; 2-heating tube ; 3 -stemming materials ; 4-shear sheet ; 5 - energy drain head ; 6- liquid storage pipe ; 7-rock mass; 8 - gaseous carbon dioxide

Figure 1: Principle of carbon dioxide phase transition fracturing.

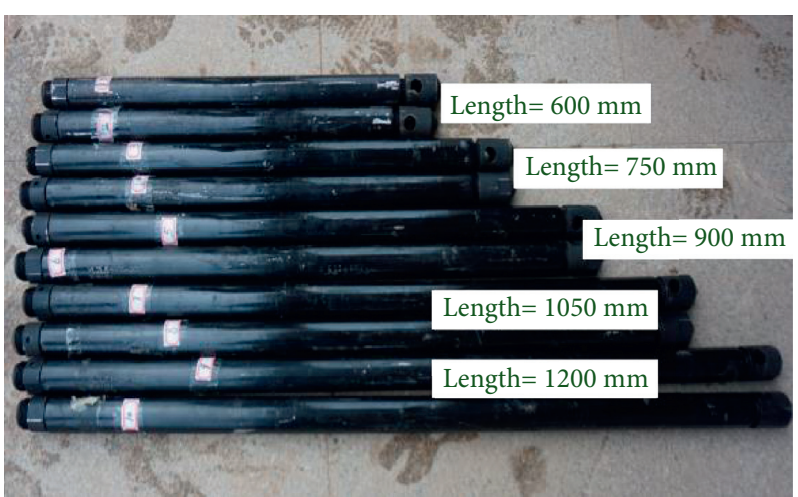

(a)

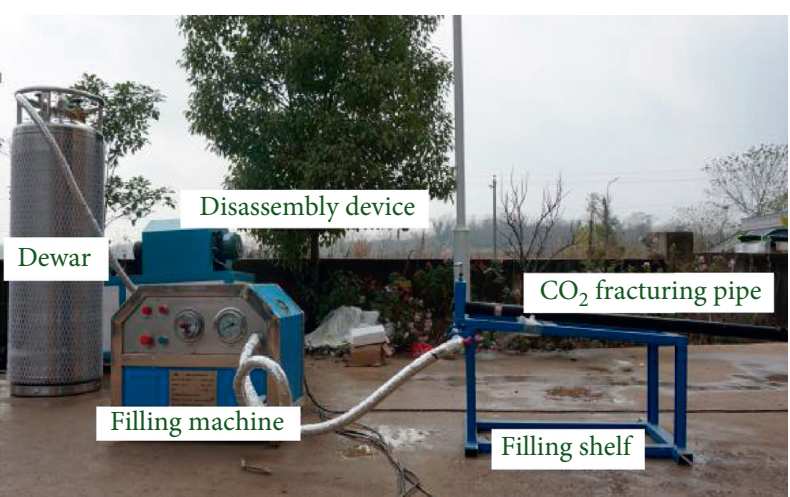

(b)

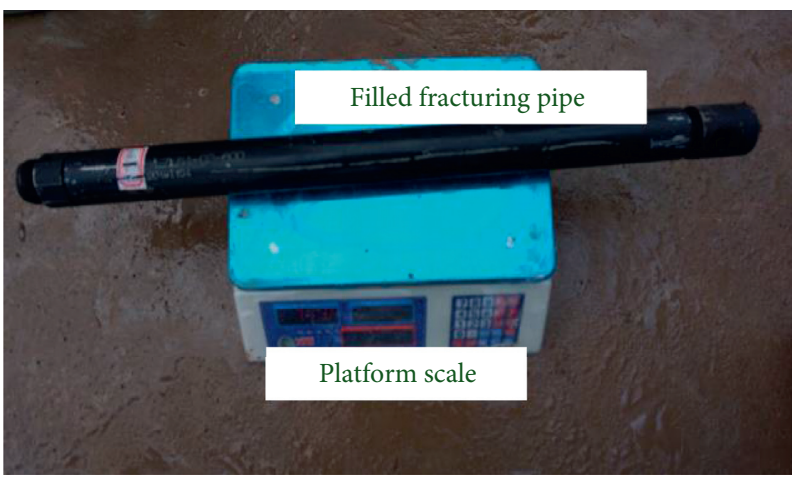

(c)

FIGURE 2: Equipment of carbon dioxide phase transition fracturing. (a) $\mathrm{CO}_{2}$ fracturing pipes with different capacities. (b) Filling equipment of $\mathrm{CO}_{2}$ fracturing pipe. (c) Platform scale for measuring the filled fracturing pipe.

pipe from flying. Despite the fact that the drill hole was stemmed, we also connected the steel strand to the lifting head for double protection of the fracturing pipe. The heating tube is then connected, the fuse is led to a safe range, and five fracturing pipes of different filling amounts were detonated in sequence.
Moreover, since too little carbon dioxide mass and too thick the shear sheet may cause the fracturing pipe to fail to detonate, a test fracturing with $460 \mathrm{~g}$ carbon dioxide and $3.5 \mathrm{~mm}$ shear sheet was carried out before the formal tests to explore whether the fracturing pipe in this situation can be detonated. The results showed that the $3.5 \mathrm{~mm}$ shear sheet 
could be destroyed even with the smallest capacity fracturing pipe. Therefore, the $\mathrm{CO}_{2}$ filling amounts in these tests were $460 \mathrm{~g}, 585 \mathrm{~g}, 760 \mathrm{~g}, 870 \mathrm{~g}$, and $960 \mathrm{~g}$, respectively. Furthermore, among all the tests, the shear sheet thickness was $3.5 \mathrm{~mm}$, and the drill hole depth and diameter were $900 \mathrm{~mm}$ and $115 \mathrm{~mm}$, respectively.

3.2. Test Results. After the fracturing pipe was detonated, a large amount of fog remained in the fracturing crater, which is due to the vaporization and heat absorption of liquid carbon dioxide, leading to a decrease in the surrounding temperature and the condensation of water vapor in the air into small water droplets and the formation of fog. Moreover, the morphology for $\mathrm{CO}_{2}$ phase transition crater is significantly different from blasting, and the crater shape is longer in jet direction. The reason is that the high-pressure carbon dioxide can only be released from two symmetrical release holes due to the special structure for fracturing pipe, resulting in a Mohaupt effect in the jet direction. The load in the jet direction is much higher than that of other directions; the surrounding rock in this direction is firstly damaged induced the dynamic stress, resulting in a large number of initial cracks. Then, the high-pressure $\mathrm{CO}_{2}$ expands into the initial cracks, leading them to continue to propagate. Since the borehole in jet direction firstly undergoes the jet, the corresponding axis length for $\mathrm{CO}_{2}$ phase transition crater was longer, as is shown in Figure 3. By contrast, the blasting crater follows the Livingston blasting crater principle, and because the blasting energy is released in every direction, there are all the circular craters; the surrounding rocks in all directions get the same degree of fragmentation.

To quantitatively analyse the influence of different $\mathrm{CO}_{2}$ filling amounts on rock fragments, the plane perpendicular to the axis of the drill hole was chosen as the reference plane. The debris in the fracturing crater was cleaned up; the distance between the contour of the crater and the reference plane is measured on a $100 \mathrm{~mm} \times 100 \mathrm{~mm}$ grid to find out the crater depth at each measuring point. The area $S_{i}$ of each cross section of fracturing crater was calculated through the Simpson equation, and the crater volume $V$ was obtained by the prism volume formula:

$$
\begin{aligned}
& S_{i}=\frac{B}{3}\left[\left(Y_{0}+Y_{n}\right)+2\left(Y_{2}+Y_{4}+\cdots+Y_{2 i}+\cdots\right)+4\left(Y_{1}+Y_{3}+\cdots+Y_{2 i+1}+\cdots\right)\right], \\
& V=\frac{B}{3}\left[\left(s_{1}+s_{n}\right)+2\left(s_{1}+s_{2}+\cdots+s_{i}+\cdots s_{n}\right)+\sum_{i=1}^{n} \sqrt{s_{i} s_{i+1}}\right]
\end{aligned}
$$

where $S_{i}$ is the area of the crater cross section, $\mathrm{m}^{2} ; B$ is the horizontal distance between two adjacent measured points, $B=0.1 \mathrm{~m}$; and $Y_{i}$ is the fracturing crater depth of measuring point $i, \mathrm{~m}$.

After cleaning up the broken rocks in the fracturing crater, the boundary of the fracturing crater was determined. Since the $\mathrm{CO}_{2}$ phase transition fracturing crater is elliptical, taking the drill hole as the center, the crater diameter is measured at $45^{\circ}$ intervals. The length of the long axis and the short axis of the ellipse were counted, as Figure 4 shows.

From Figure 4, it can be seen that as the $\mathrm{CO}_{2}$ filling amount increases, the long axis length of the fracturing crater gradually increases, but the short axis length first increases and then decreases. With the $\mathrm{CO}_{2}$ filling amount increasing, the crater volume gradually grew; the $\mathrm{CO}_{2}$ powder factor remained unchanged within a certain filling amount, beyond which it increases significantly, as shown in Figure 5. When the $\mathrm{CO}_{2}$ filling amount increases from $460 \mathrm{~g}$ to $970 \mathrm{~g}$, even if the growth rate of crater volume slows down, its value gradually increases. It can also be found that after the $\mathrm{CO}_{2}$ filling amount exceeds a certain value, its influence on the crater volume growth becomes smaller but becomes significantly larger on the powder factor. The reason for that is that the $\mathrm{CO}_{2}$ filling amount is not the only factor that affects the crater volume; the shear sheet thickness limits the peak pressure in $\mathrm{CO}_{2}$ phase transition fracturing and also controls the rock fragmentation. While the peak pressure is constant, if only the $\mathrm{CO}_{2}$ filling amount increases, there is a clear threshold for fracturing crater volume. When the crater volume reaches the threshold value, it is meaningless to further increase the filling amount, which will only increase the cost of construction and reduce the economic efficiency. To better improve the energy utilization ratio of rock fragmentation, it is necessary to further explore the influence of shear sheet thickness on rock fragmentation.

\section{Effect of Shear Sheet Thickness on Rock Fragments}

4.1. Field Test. It is acknowledged that the shear sheet is the most important component to influence the peak shock force. When the absolute pressure in fracturing pipe exceeds the shear sheet strength, high-pressure carbon dioxide gas will be ejected through the energy drain head [20]. The thickness of the shear sheet is the determining factor in determining its failure pressure, and it is generally accepted that the thicker the shear sheet, the higher the corresponding failure pressure. To control the thickness as a single variable, all shear sheets were made by Q235a steel in these tests. Shear sheets with thicknesses of $1.5 \mathrm{~mm}, 2.0 \mathrm{~mm}, 2.5 \mathrm{~mm}$, $3.0 \mathrm{~mm}$, and $3.5 \mathrm{~mm}$ were selected; a fracturing pipe with the length of $900 \mathrm{~mm}$ was used. The $\mathrm{CO}_{2}$ filling amount for the five tests is $755 \mathrm{~g}, 760 \mathrm{~g}, 760 \mathrm{~g}, 755 \mathrm{~g}$, and $750 \mathrm{~g}$, respectively. Although there was some variation in the amount of $\mathrm{CO}_{2}$ 


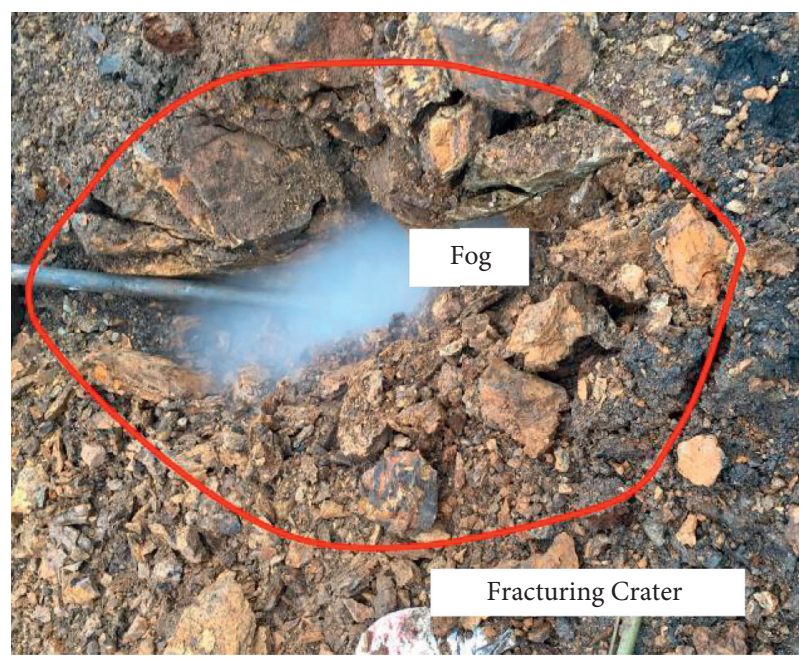

Figure 3: Shape of the fracturing crater.

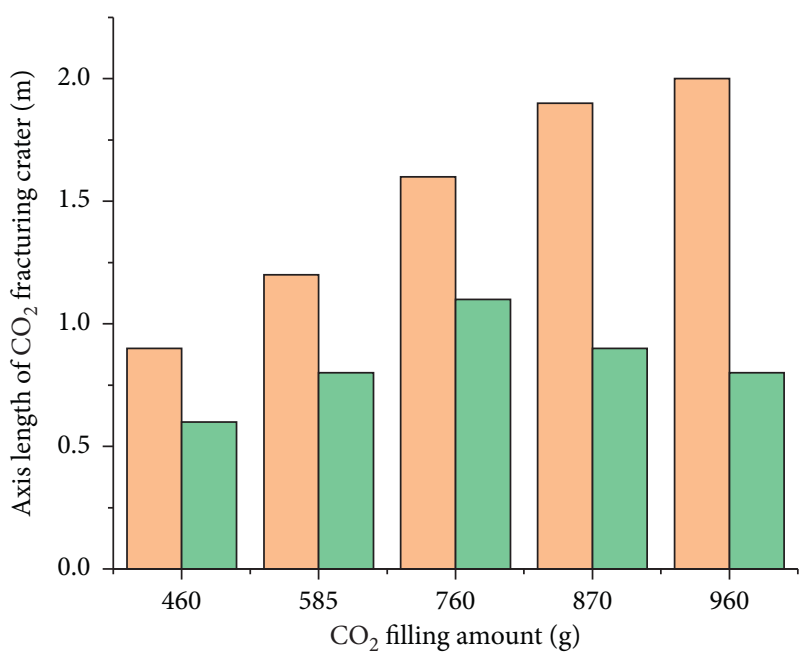

Long axis of crater Short axis of crater

FIGURE 4: Long and short axes length of $\mathrm{CO}_{2}$ fracturing crater with different $\mathrm{CO}_{2}$ filling amounts.

filled between the different pipes, this variation was within a negligible margin of error.

Different from the previous tests, since the shear sheet greatly affected the peak stress in the rock mass, the strain test was added. Considering that the higher impact appears in the jet direction, all the self-made strain testing devices were installed at a distance of $2 \mathrm{~m}$ from the drill hole in the jet direction. The strain signals at the monitoring points are collected using dynamic strain testing apparatus DH5960. The schematic sketch of strain measuring test design was shown in Figure 6; the procedure for preparing and installing self-made strain testing devices is as follows:

(1) Prepare several plain bars and iron sheets and weld three iron sheets $X, Y$, and $Z$ at the underside of the plain bar. Three iron sheets are perpendicular to each other; the sheet $Z$ has a long side in the same direction as the long axis of the plain bar.

(2) Make the gypsum strain block, pour the stirred quick-setting gypsum into the strip mold, take it out after the gypsum solidifies, and polish the block surface to make it smooth enough.

(3) Before attaching the strain gauges to the strain block, the resistance of strain gauges shall be measured; then the epoxy resin shall be used to attach the strain gauges for ensuring that the strain gauge is firmly bonded with strain block and the adhesive layer is uniform Then, paste the gypsum strain block on each iron sheet..

(4) Connect the strain gauges to the wires via terminals, and number the wires. Since the insulation resistance and bonding strength of the strain gauge will be reduced when it is affected with damp, add painted gypsum as a protective layer in the surface of strain gauges. Moreover, the above treatment can avoid damage caused by friction with the drill hole when the strain testing device is installed.

(5) Bury the strain testing device in the predrilled measuring hole, and ensure that the strain gauges are at the same depth as the gas outlet of the fracturing pipe and the $X$ sheet is pointing towards the fracturing pipe; then stem the drill hole with quicksetting gypsum.

As shown in Figure 7, when we started to conduct $\mathrm{CO}_{2}$ phase transition fracturing, the self-made strain testing device was first installed as described above, and then the fracturing pipe was buried. It should be noted that the direction of the line connecting the two symmetrical gas outlets should be in the same line with the long axis of $X$ sheet when installing the fracturing pipe.

\subsection{Test Results}

4.2.1. Peak Stress with Different Shear Sheet Thicknesses. During these tests, DH5960 super dynamic signal test system was used to record the micro strain. The DH5960 super dynamic signal test system, with $20 \mathrm{MHz}$ maximum transient sampling rate and $1 \mathrm{MHz}$ frequency response, is designed for impact and blasting test. Moreover, Gigabit Ethernet switch is adopted to expand; only one computer can also realize unlimited multichannel super dynamic signal parallel synchronous testing and analysis. To meet the dynamics requirements of strain measurement, in these tests, we set a measuring frequency of $20000 \mathrm{~Hz}$. Since the strain real-time curve in the field experiment can be interfered by a series of field factors such as electromagnetic, the WNOISE function was utilized to denoise field strain data to get more realistic strain signals. This method processes wavelet decomposition coefficient to separate signal and noise with the help of different characteristics of signal and noise in wavelet transform.

These electric resistance strain gauges only measured the strain in a certain direction at the measuring point. To obtain 


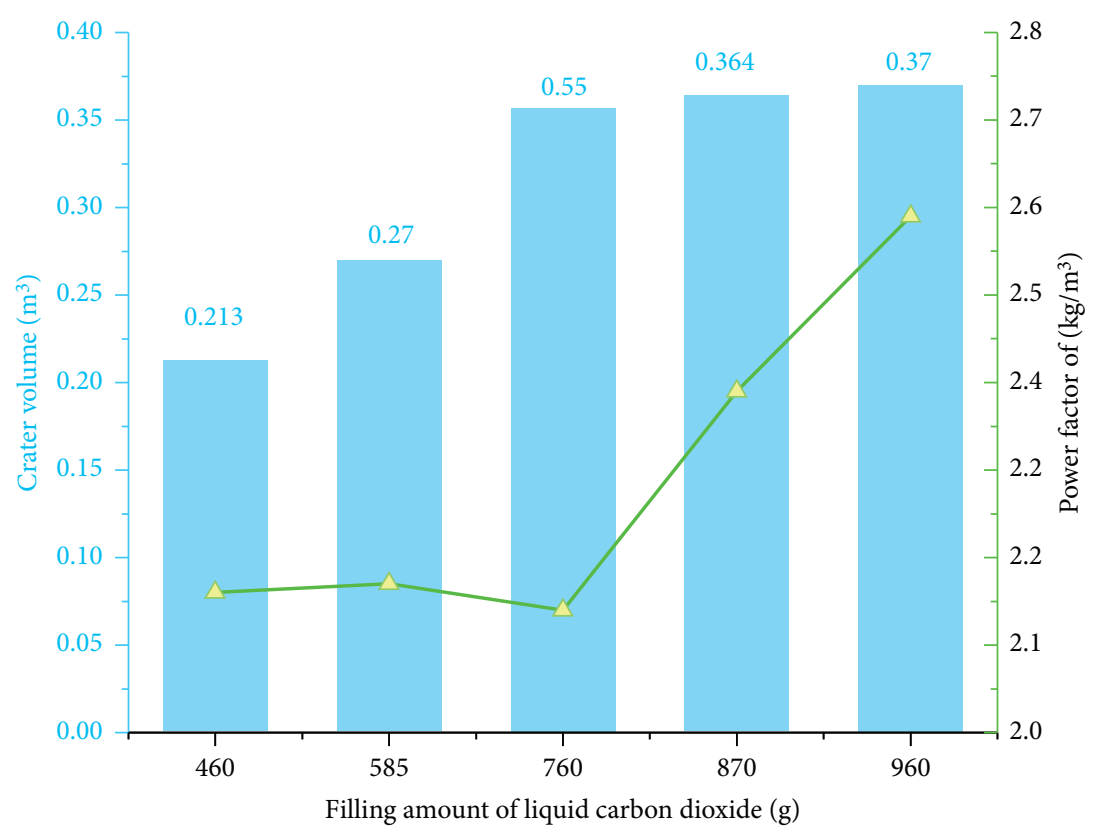

FIgURE 5: Variation of crater volume and powder factor with varying $\mathrm{CO}_{2}$ filling amounts.

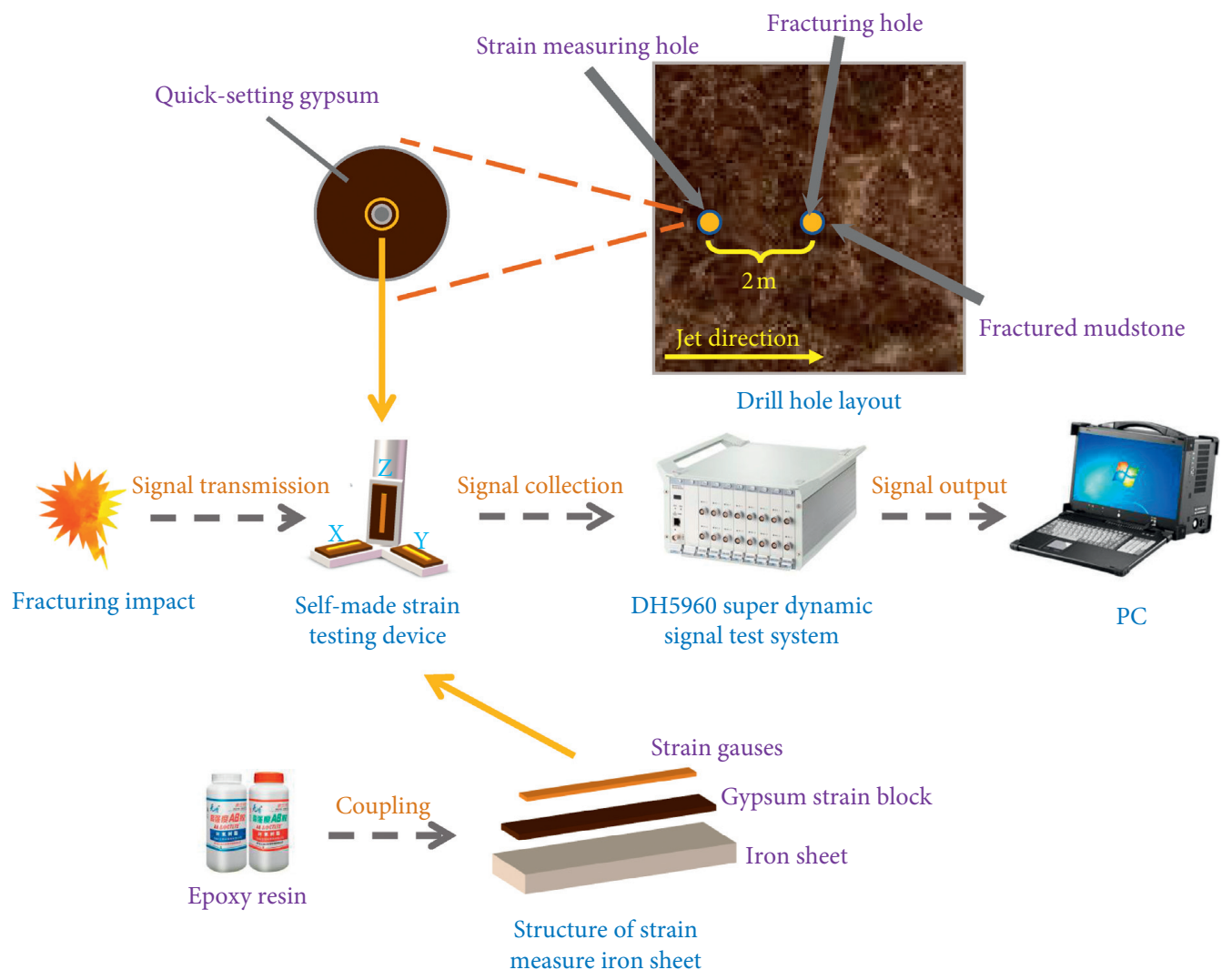

FIgURE 6: Schematic sketch of the strain measuring test design.

the peak dynamic stress, the strain value obtained needs to be converted [21-23]. Since the monitoring point was in a state of three-dimensional stress state, three perpendicular strain gauges were pasted along with the three principal stress directions. Assuming that the principal strain in the radial, tangential, and vertical directions of the measuring point is $\varepsilon_{x}, \varepsilon_{y}$, and $\varepsilon_{z}$, respectively, according to the generalized Hooke's law, the corresponding principal stresses $\sigma_{x}$, 


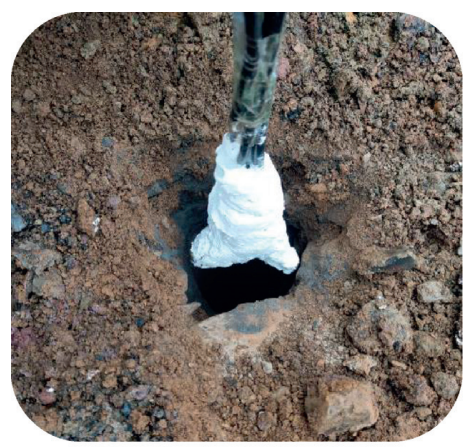

Put the self-made strain testing device into measuring hole

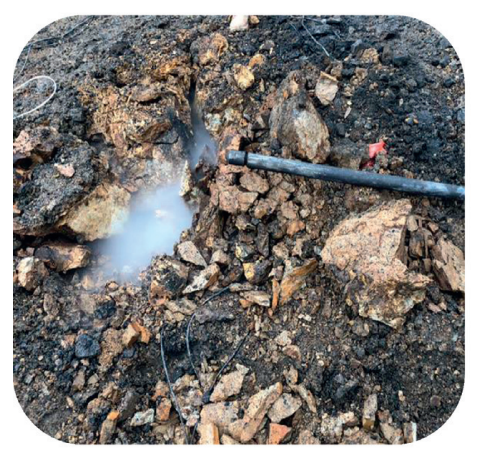

Detonate the fracturing pipe by the current
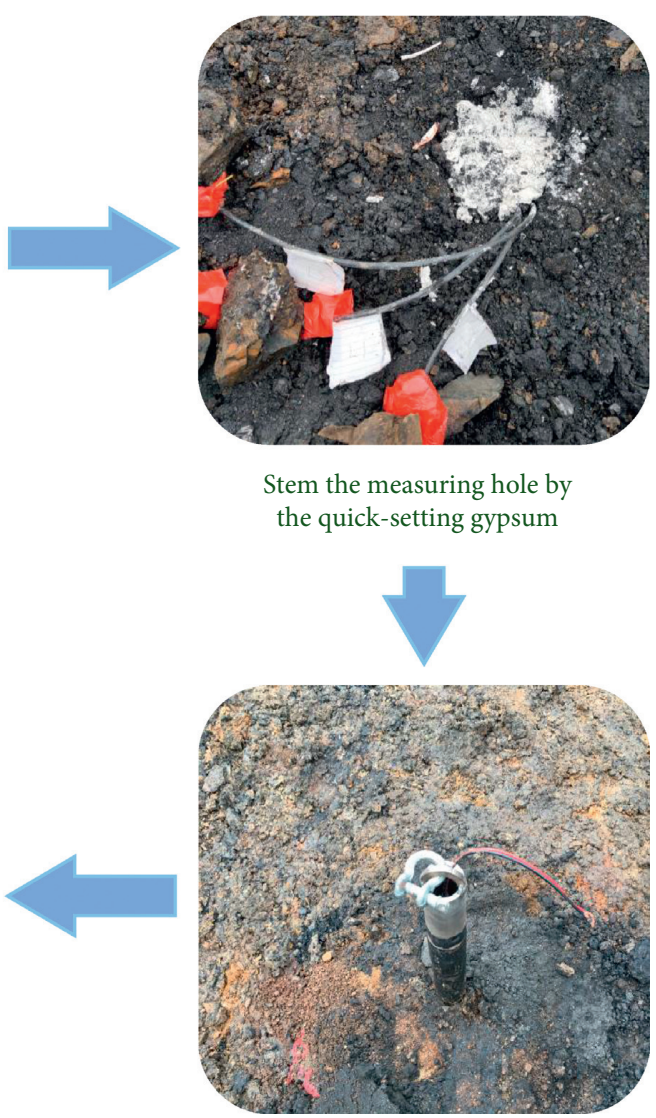

Bury the fracturing pipe into the drill hole

Figure 7: Installation process of the fracturing pipe and strain testing device.

$\sigma_{y}$, and $\sigma_{z}$ of radial, tangential, and vertical direction are as follows:

$$
\left\{\begin{array}{l}
\sigma_{x}=\frac{E}{1+v}\left(\frac{v}{1-2 v} \theta+\varepsilon_{x}\right) \\
\sigma_{y}=\frac{E}{1+v}\left(\frac{v}{1-2 v} \theta+\varepsilon_{y}\right), \\
\sigma_{z}=\frac{E}{1+v}\left(\frac{v}{1-2 v} \theta+\varepsilon_{z}\right)
\end{array}\right.
$$

where $\theta$ is the volumetric strain, $\theta=\varepsilon_{x}+\varepsilon_{y}+\varepsilon_{y}, E$ is the dynamic modulus of elasticity, and $v$ is the dynamic Poisson's ratio.

To investigate the relationship between the shear sheet thickness and the peak stress, the peak strains of five tests were recorded; then the peak strain in each test is converted to peak stress by equation (3), as shown in Figure 8 .

From Figure 8, it can be seen that when the shear sheet thickness is the same, the peak stress in the vertical direction (Z-direction) of the measuring point is the largest, and the peak stress in the radial ( $X$-direction) and tangential ( $Y$ direction) directions does not differ much, indicating that the dominant stress direction is the vertical direction. As the

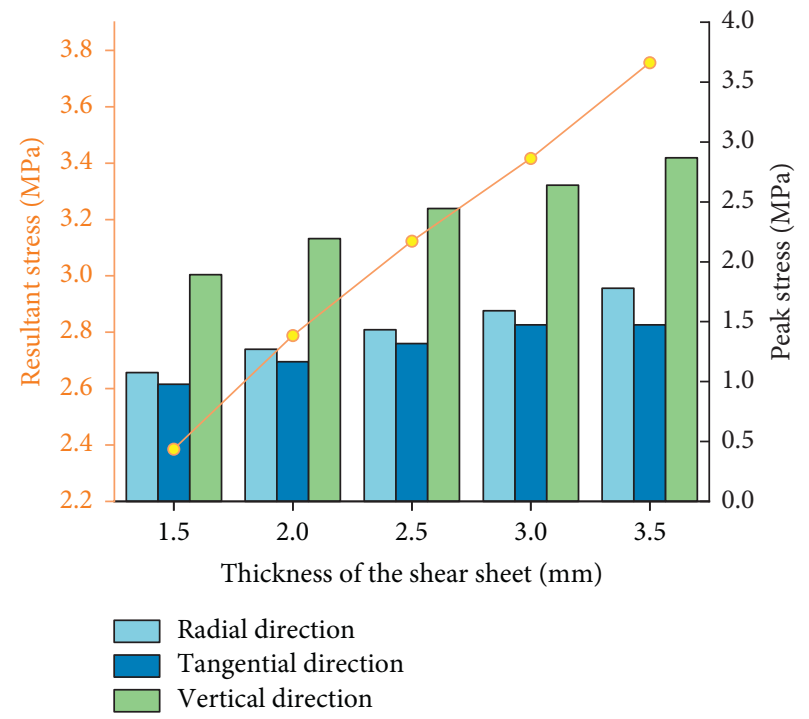

Figure 8: Variation of peak stress in different directions and resultant stress with varying thickness of the shear sheet.

shear sheet thickness increases, the resultant stress also grows continuously. When the shear sheet thickness varies from $1.5 \mathrm{~mm}$ to $3.5 \mathrm{~mm}$, the resultant stress increases by $1.371 \mathrm{MPa}$. It can be seen that the peak stress increases with 


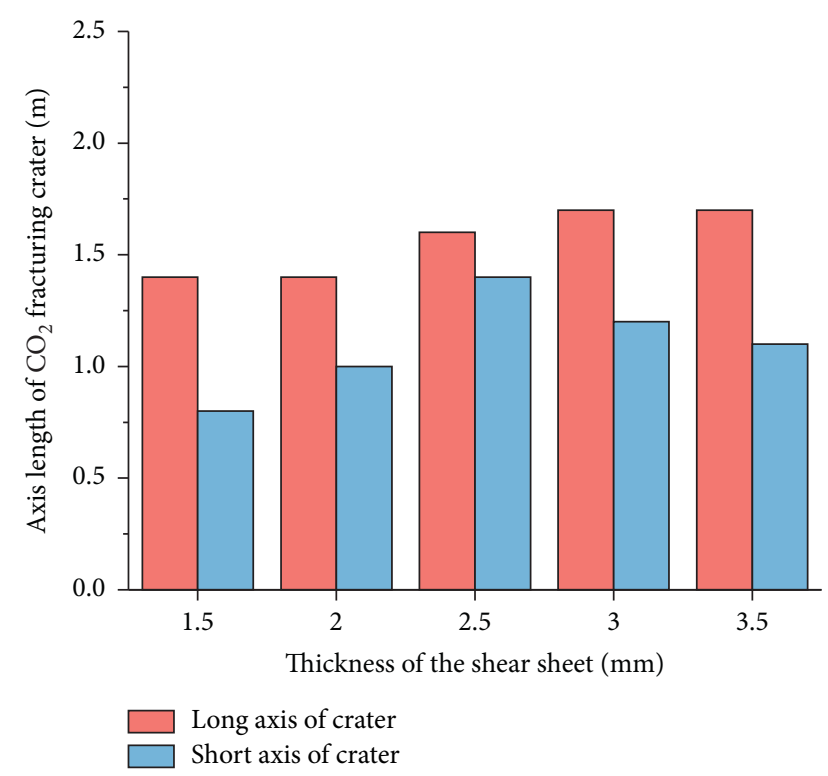

FIGURE 9: Long and short axes length of $\mathrm{CO}_{2}$ fracturing crater with different thickness of shear sheet.

the growth of the shear sheet strength, and its growth rate is unchanged. The measured peak stress can reflect the peak stress of the drill hole wall in the direction of the air outlet of the fracturing. It is indicated that the thicker the shear sheet, the larger the peak stress.

4.2.2. Crater Volume with Different Shear Sheet Thicknesses. The previous measuring method for crater volume and crater morphology was utilized in the tests with variable shear sheet thickness. Furthermore, the $\mathrm{CO}_{2}$ powder factors were obtained by these five tests. The results are shown in Figures 9 and 10.

It can be seen that as the shear sheet thickness increases, the crater volume and long axis length of the fracturing crater gradually increase, the $\mathrm{CO}_{2}$ powder factor decreases, while the length of the short axis first grows and then declines. Moreover, when the $\mathrm{CO}_{2}$ filling amount is equivalent in these five tests, the peak stress in the jet direction gradually increases with the shear sheet thickness increases, resulting in the growth of the effective damage range of the rock mass.

When the shear sheet thickness varies from $1.5 \mathrm{~mm}$ to $2.5 \mathrm{~mm}$, the crater volume increases by $0.059 \mathrm{~m}^{3}$ and the powder factor decreases by $1.19 \mathrm{~kg} / \mathrm{m}^{3}$. As the shear sheet thickness is changed from $2.5 \mathrm{~mm}$ to $3.5 \mathrm{~mm}$, the crater volume increases by $0.132 \mathrm{~m}^{3}$, and the powder factor decreases by $1.30 \mathrm{~kg} / \mathrm{m}^{3}$. It is indicated that with the increase of shear sheet thickness, the growth rate of crater volume increases, but the decline rate of powder factor remains unchanged.

Comparing the test results for different $\mathrm{CO}_{2}$ filling amount levels and different shear sheet thickness, it can be seen that the crater volume changes with variable $\mathrm{CO}_{2}$ filling are smaller than the crater volume with variable shear sheet thicknesses. Moreover, a conclusion can be drawn from the

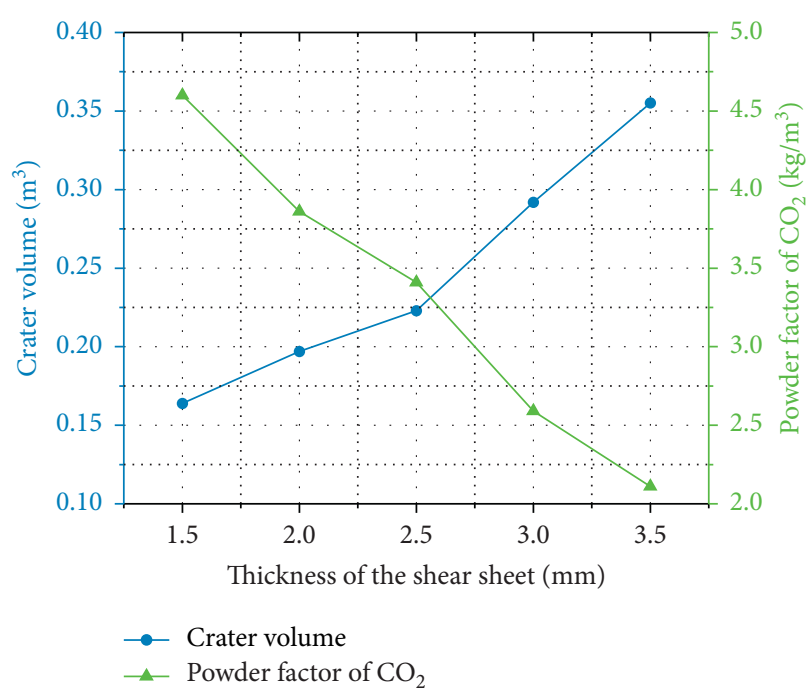

FIgURE 10: Crater volume and powder factor of $\mathrm{CO}_{2}$ with variable shear sheet thickness.

evaluation indicators, such as powder factor and crater radius, that the effect of shear sheet thickness on the rock fragments is obviously larger than the amount of liquid carbon dioxide.

\section{Conclusions}

In this paper, the fracturing craters volume were counted, and the effects of $\mathrm{CO}_{2}$ filling amount and shear sheet thickness on rock fragments were investigated through the in situ rock breaking test. Moreover, the dynamic strain test under the impact of $\mathrm{CO}_{2}$ phase transition fracturing was carried out. The following conclusions are obtained:

(1) The fracturing crater of $\mathrm{CO}_{2}$ phase transition fracturing is significantly different from the blasting and is longer in the jet direction and shorter in the vertical jet direction. It is shaped like an ellipsoidal cone.

(2) As the $\mathrm{CO}_{2}$ filling amount increases, the crater volume gradually increases, and its growth rate gradually decreases. The powder factor of $\mathrm{CO}_{2}$ remains constant within a certain $\mathrm{CO}_{2}$ filling amount and increases significantly beyond that value. The length of the long axis of the crater gradually increases with the increasing $\mathrm{CO}_{2}$ filling amounts, but the length of the short axis first grows and then decreases.

(3) When the shear sheet thickness increases, the corresponding resultant stress grows, but the grow slope is constant. Even if the powder factor decreases, the value of crater volume and its growth rate continually increase with the growing shear sheet thickness. The change pattern of long and short axis length in variable shear sheet thickness is the same as that in variable $\mathrm{CO}_{2}$ filling amounts. Overall, shear sheet thickness has a greater effect on rock fragmentation than $\mathrm{CO}_{2}$ filling amount. 
Although the change characteristics of the strains we measured are consistent with the existing understanding, it should be noted that the above conclusions were obtained by a limited number of tests. To get a more universal statistical law, more tests in different fractured rock masses need to be carried out in the future. The influence of rock properties and confining pressures on rock fragmentations should be further explored in fractured rock masses, which is significant for rock excavation in deep rock engineering.

\section{Data Availability}

The data used to support the findings of this study are included within the article.

\section{Conflicts of Interest}

The authors declare that there are no conflicts of interest regarding the publication of this study.

\section{Acknowledgments}

This study was sponsored by the National Natural Science Foundation of China (Grant nos. 42072309, 41807265, and 41972286) and the Hubei Key Laboratory of Blasting Engineering Foundation (Grant nos. HKLBEF202002 and HKLBEF202001).

\section{References}

[1] T. S. Bajpayee, T. R. Rehak, G. L. Mowrey, and D. K. Ingram, "Blasting injuries in surface mining with emphasis on flyrock and blast area security," Journal of Safety Research, vol. 35, no. 1, pp. 47-57, 2004.

[2] N. Jiang, B. Zhu, X. He, C. Zhou, X. Luo, and T. Wu, "Safety assessment of buried pressurized gas pipelines subject to blasting vibrations induced by metro foundation pit excavation," Tunnelling and Underground Space Technology, vol. 102, Article ID 103448, 2020.

[3] T. Caldwell, A Comparison of Non-explosive Rock Breaking Techniques, The University of Queensland, Brisbane, Australia, 2004.

[4] S. Zhou, N. Jiang, X. He, and X. Luo, "Rock breaking and dynamic response characteristics of carbon dioxide phase transition fracturing considering the gathering energy effect," Energies, vol. 13, no. 6, p. 1336, 2020.

[5] N. Vidanovic, S. Ognjanovic, and N. Ilincic, "Application of unconventional methods of underground premises construction in coal mines," Technics Technologies Education Management, vol. 6, no. 4, pp. 861-865, 2011.

[6] X. Xie, X. Li, Q. Lii, and H. Ma, "Liquid $\mathrm{CO}_{2}$ phase-transforming rock fracturing technology in pile-well excavation," Journal of Central South University, vol. 49, no. 8, pp. 20312038, 2018.

[7] T. Lu, Z. Wang, H. Yang, P. Yuan, Y. Han, and X. Sun, "Improvement of coal seam gas drainage by under-panel cross-strata stimulation using highly pressurized gas," International Journal of Rock Mechanics and Mining Sciences, vol. 77, pp. 300-312, 2015.

[8] Y. Fan, B. Qin, Q. Zhou, Q. Shi, D. Ma, and J. Wu, "Liquid $\mathrm{CO}_{2}$ phase transition fracturing technology and its application in enhancing gas drainage of coal mines," Adsorption Science and Technology, vol. 38, no. 9-10, pp. 393412, 2020.

[9] H. Chen, Z. Wang, L. Qi, and F. An, "Effect of liquid carbon dioxide phase change fracturing technology on gas drainage," Arabian Journal of Geosciences, vol. 10, no. 14, pp. 1-9, 2017.

[10] Z. Nie, "Application of cardox dioxide blasting technique in coal mines," Coal Technology, vol. 26, no. 8, pp. 36-37, 2007.

[11] Z. Du and X. Yufei, "Study on carbon dioxide blasting mining and driving equipment and technology," Coal Science and Technology, vol. 44, no. 9, pp. 36-42, 2016.

[12] Y. B. Zhang, E. Q. Li, J. W. Liu, X. J. Leng, and W. G. Li, "Applications of carbon dioxide cannon blasting on the problem of triangular flap top in coal mine handling mechanized mining face," Applied Mechanics and Materials, vol. 256-259, pp. 71-74, 2012.

[13] S. P. Singh, "Non-Explosive applications of the PCF concept for underground excavation," Tunnelling and Underground Space Technology, vol. 13, no. 3, pp. 305-311, 1998.

[14] D. S. Zhan, Carbon Dioxide Filling Capacity and Cracking Effect Simulation Analysis, China Coal Research Institute, Beijing, China, 2017.

[15] Y. Zhang, J. Deng, B. Ke, H. Deng, and J. Li, “Experimental study on explosion pressure and rock breaking characteristics under liquid carbon dioxide blasting," Advances in Civil Engineering, vol. 2018, Article ID 7840125, 2018.

[16] Y. Zhang, J. Deng, H. Deng, and B. Ke, "Peridynamics simulation of rock fracturing under liquid carbon dioxide blasting," International Journal of Damage Mechanics, vol. 28, no. 7, pp. 1038-1052, 2018.

[17] M. Tao, H. T. Zhao, X. B. Li, and A. Ma, "Comprehensive comparative analysis of liquid $\mathrm{CO}_{2}$ phase change fracturing and explosive rock fracturing," Blasting, vol. 35, no. 2, pp. 41-49, 2018.

[18] Z. C. Tian, Crack Form and Influencing Factors of Liquid Carbon Dioxide Phase Transition Fracturing, China University of Mining and Technology, Xuzhou, China, 2018.

[19] X. H. Zhou, J. L. Men, and D. P. Song, "Research on optimal borehole parameters of antireflection in coal seam by liquid $\mathrm{CO}_{2}$ blasting," Chinese Journal of Rock Mechanics and Engineering, vol. 35, no. 3, pp. 524-529, 2106.

[20] K. P. Zhou, B. Ke, J. L. Li, Y. N. Zhang, and L. Cheng, "Pressure dynamic response and explosion energy of liquid carbon dioxide blasting system," Blasting, vol. 34, no. 3, pp. 7-13, 2017.

[21] J. Y. Li, Experimental Study on the Morphology of Blasting Crater with Different Strength Concrete, Southwest University of science and technology, Mianyang, China, 2018.

[22] H. B. Chu, X. L. Yang, A. J. Hou, and Y. Yu, "A simulationbased experimental study on explosion stress wave propagation and attenuation in coal," Explosion and Shock Waves, vol. 32, no. 2, pp. 185-189, 2012.

[23] J. P. Zhao, "Experiment study on measurement and separation of explosive wave signals near blast hole in concrete," Blasting, vol. 29, no. 2, pp. 4-9+18, 2012. 